\title{
Demographic, laboratory and clinical characterisation of adult portuguese asthmatic patients
}

\author{
O. Lourenço ${ }^{a}$, A.M. Fonseca ${ }^{a}$ and L. Taborda-Barata ${ }^{a, b}$ \\ ${ }^{a}$ CICS, Centro de Investigação em Ciências da Saúde. University of Beira Interior. Covilhã. Portugal. ' ${ }^{\text {Cova da }}$ \\ Beira Hospital. Covilhã. Portugal.
}

\begin{abstract}
Background: Asthma is a heterogeneous chronic inflammatory condition characterised by reversible airway obstruction and hyperresponsiveness associated with underlying bronchial inflammation and structural changes. It represents an increasing health problem and is a huge burden on the patients, their families and society. The aim of the study was to characterise the adult asthmatic population attending a Hospital Allergy Clinic between the years of 2003 and 2006.

Methods: Clinical files from the Allergy Outpatient Clinic of Cova da Beira Hospital were sequentially studied. The total population analysed included 335 female and 130 male asthmatic patients. Bronchial asthma was characterised by clinical history, skin prick testing to aeroallergens, determination of total and specific $\lg E$ and lung function testing, and classified according to international guidelines.

Results: Of the patients studied, $70 \%$ had allergic asthma, and $30 \%$ had non-allergic asthma. When
\end{abstract}

\section{Correspondence:}

Professor Luis Taborda-Barata, M.D., Ph.D.

Department of Medical Sciences.

Faculty of Health Sciences

University of Beira Interior

Avda. D. Afonso Henriques

6200-506 Covilhã. Portugal

Tel: + 351 275329002/3

Fax: + 351275329099

E-mail: tabordabarata@fcsaude.ubi.pt compared to allergic asthma, non-allergic asthma was more frequently associated with older age, perennial symptoms and female gender. More allergic than non-allergic asthma patients also had rhinitis and the reverse was true regarding drug allergy and oesophageal reflux. Grass pollen and mites were the major sensitisers for allergic asthmatics. The sensitisation profile was significantly different between urban- and rural-based asthmatic patients regarding tree pollen, fungi and moulds.

Conclusions: In this population, rhinitis was more frequently associated with allergic than with non-allergic asthma. The two types of asthma did not differ in clinical severity or changes in lung function. Sensitisation profiles were different between the urban and rural patients.

Key words: Allergens. Asthma. Human. Prevalence. Rural. Urban

\section{INTRODUCTION}

Asthma is a chronic, complex, obstructive lung disease characterised by acute symptomatic episodes of varying bronchial constriction that occur in response to allergens or other triggers such as viral infections and exercise. Asthma is a major cause for work and school absenteeism with repercussions on quality of life and high socio-economic impact ${ }^{1,2}$.

The majority of bronchial asthma cases generally start in childhood or adolescence in individuals responding to common aeroallergens and is mediated by immunologic mechanisms (allergic asthma). Other patients develop asthma later in life, often as a 
consequence of viral respiratory infections and without history or symptoms characteristic of atopy or allergic diseases (non-allergic asthma) ${ }^{3}$. Most patients with allergic asthma have other concurrent allergic diseases, namely rhinitis, which should be treated in order to improve asthma symptoms. In addition to phenotypes, asthma can also be classified according to its severity ranging from intermittent, mild transient episodes to severe, chronic, life-threatening bronchial obstruction ${ }^{5,6}$.

Asthma is a worldwide disease that has been recognized for centuries, but prevalence figures vary, in part, because of differences in definition and methods of case finding. In order to know the prevalence of allergic disease worldwide, the International Study of Asthma and Allergies in Childhood (ISAAC) has been developing for the last 15 years, involving more than 50 countries. Portuguese data estimate a prevalence of actual asthma of $12.9 \%$ in 6-7 year old and of $21.8 \%$ in $13-14$ year old 7,8 . The data from the European Community Respiratory Health Survey (ECRHS), which involved surveys of asthma and allergic rhinitis prevalence in adults aged 20-44 years, estimated that $5 \%$ of the Portuguese adult population has bronchial asthma ${ }^{9}$. However, in spite of these aspects, studies describing the features of Portuguese asthma patients are lacking in the literature.

To increase the current knowledge about asthma patients in Portugal we aimed at characterising the adult asthmatic population attending the Allergy Clinic of the Cova da Beira Hospital between the years of 2003 and 2006.

\section{MATERIALS AND METHODS}

Clinical files from patients attending the Allergy Outpatient Clinic of the Cova da Beira Hospital between 2003 and 2006 were sequentially studied.

The study protocol was approved by the Hospital Ethics Committee.

The diagnosis of bronchial asthma was based upon clinical history, physical examination, pulmonary function tests, and response to inhaled $\beta$-adrenergic agents according to international guidelines ${ }^{5}$. Skin prick testing to aeroallergens and determination of total and specific IgE were also performed on the asthmatic patients.

The assessment of severity was based on daytime symptoms, night-time symptoms, frequency and intensity of attacks, impact on daily activities, asthma treatment used and predicted percentages of forced expiratory volume in one second (FEV1) and peak expiratory flow (PEF). All patients were also examined by anterior rhinoscopy and filled in a questionnaire regarding nasal symptoms. Smoking habits were recorded as pack-years.

The designation of "non-allergic" was applied when the history, skin prick testing, and serum specific IgE measurements included in the allergy examination were all negative.

All the data were analysed using non-parametric tests. Results are expressed as medians and range. Wilcoxon signed rank test was used for comparisons within groups and Mann Whitney U test, chi-square test and Kruskal-Wallis test were used for comparisons between groups. The association of various clinical parameters was analysed using the Spearman rank correlation test. A $p$ value of less than 0.05 was considered significant. All analyses were performed using Minitab 14 for Windows.

\section{RESULTS}

\section{Demographic characterisation}

The total population analysed included 1078 clinical files. From those, we excluded all patients under 18 years and patients not currently living in the area. In addition, 25 patients were excluded because of discordance between skin prick tests and specific lgE tests.

Four hundred and sixty five clinical files, from adult Caucasian asthmatic patients (335 females) living in the Cova da Beira area were included for further study.

\section{Allergic versus non-allergic asthmatics}

Seventy percent (327) of the asthmatic patients had a diagnosis of allergic asthma and thirty percent had non-allergic asthma. Demographically, there were significant differences between the two groups. There were more women among non-allergic asthmatics than among allergic asthmatics, with female: male ratios of 5.3 and 2.0, respectively $(p<0.001)$. In addition, allergic patients were significantly younger than non-allergic asthmatics (median age 35 [18-85] vs 51 [18-84] years, p < 0.001). Smoking habits were not different between allergic and non-allergic patients.

Bronchial asthma was classified according to the old practice that divided it into seasonal and perennial. In this regard, we observed that seasonal symptoms were more common in allergic than in non-allergic patients (40 \% versus $18.2 \%, \chi^{2}=8.992$, $p=0.003$ ). 

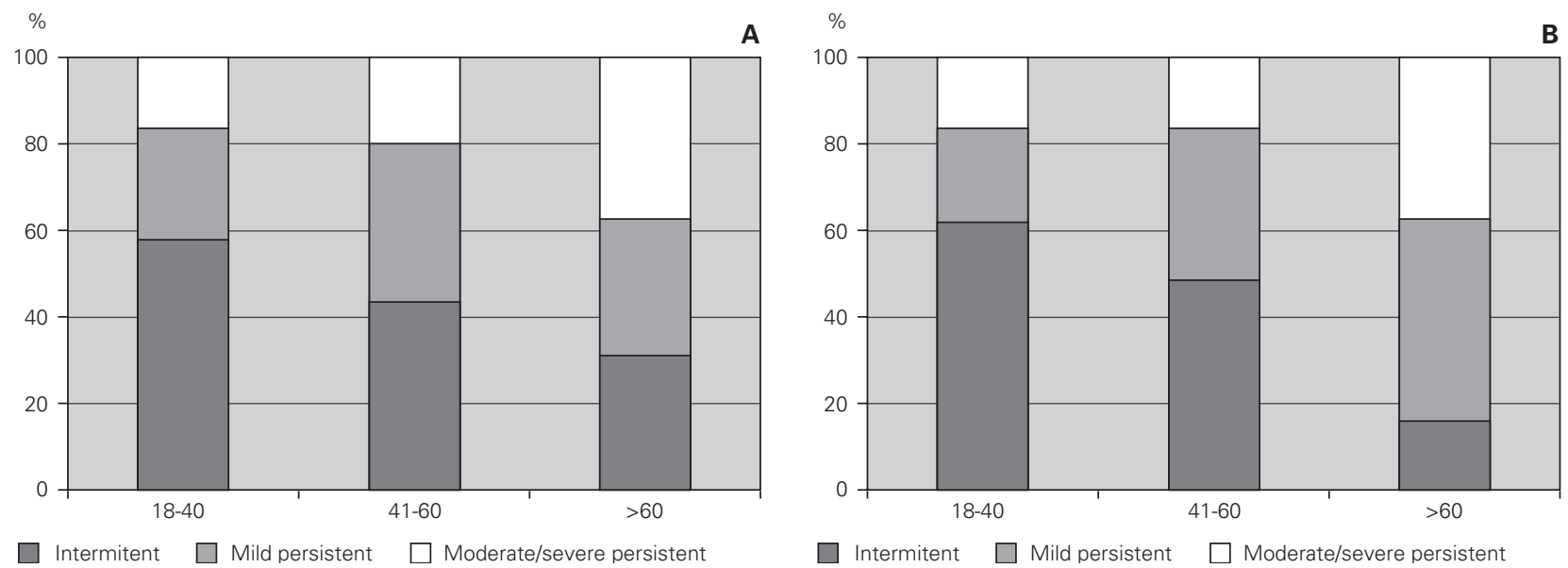

Figure 1.-Relationship between severity of asthma and age in allergic asthmatic (Panel A) and non-allergic asthmatic (Panel B) patients. Patients were grouped into three age groups and severity was assessed according to GINA guidelines. Severity increased with age but was not statistically different between the two groups.

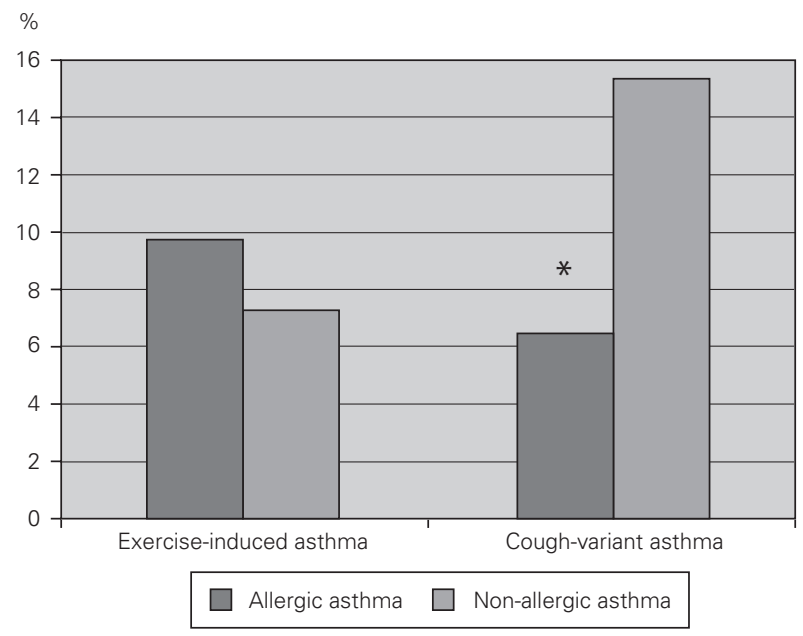

Figure 2.-Prevalence of exercise-induced and cough-variant asthma in allergic (dark columns) and non-allergic (light columns) asthmatic patients. Non-allergic asthmatics had a higher prevalence of cough-variant asthma compared to allergic asthmatics.

Patients were divided into three age groups and severity was classified according to the Global Initiative for Asthma guidelines ${ }^{6}$. With increasing age, there was an increase in the severity of asthma both in allergic and non-allergic asthmatics $\left(\chi^{2}=12.305\right.$ for allergic and 12.937 for non-allergic, $p=0.015$ and 0.012 respectively). However, there were no differences in terms of severity between allergic and non-allergic asthmatics ( $p>0.05$ ) (fig. 1).

Some patients with asthma have chronic cough as their main, if not only, symptom, and others have physical activity as the single cause for asthma. More non-allergic than allergic asthmatic patients had cough-variant asthma ( $15.2 \%$ vs $6.4 \% ; \chi^{2}=9.137$; $\mathrm{p}=0.003)$, which could not be attributed to differ-
Table I Lung function testing values in allergic and non-allergic
asthmatics

\begin{tabular}{lllc}
\hline & Allergic asthma & Non-allergic asthma & $p$ \\
\hline \% FEV1 & $101.9 \pm 1.1$ & $101.9 \pm 1.8$ & 0.6979 \\
& $102.0[51-148]$ & $103.0[49-143]$ & \\
\% FVC & $105.8 \pm 1.0$ & $107.7 \pm 1.7$ & 0.1811 \\
& $106.5[50-159]$ & $108.0[56-145]$ & \\
FEV1/FVC & $0.82 \pm 0.01$ & $0.80 \pm 0.01$ & 0.3034 \\
& $0.82[0.46-1.1]$ & $0.81[0.51-1.2]$ & \\
\% PEF & $92.7 \pm 1.2$ & $95.8 \pm 2.0$ & 0.1126 \\
& $92.0[41-151]$ & $94.5[30-155]$ & \\
\% FEF $25-75$ & $80.9 \pm 1.9$ & $78.4 \pm 3.0$ & 0.7321 \\
& $81.0[15-180]$ & $83.0[13-150]$ & \\
\hline
\end{tabular}

Data are represented as mean \pm standard error of the mean and median and range; comparisons between both groups were evaluated by the Mann-Whitney U test.

ences in work problems or exposure. Exercise-induced asthma was equally prevalent in both groups (fig. 2).

Measurements of lung function provide an assessment of the severity, reversibility, and variability of airflow limitation, and help to confirm the diagnosis of asthma. Pulmonary function test data was expressed in terms of percentage of predicted values.

Lung function testing values were not significantly different between allergic and non-allergic asthmatic patients (table I). 


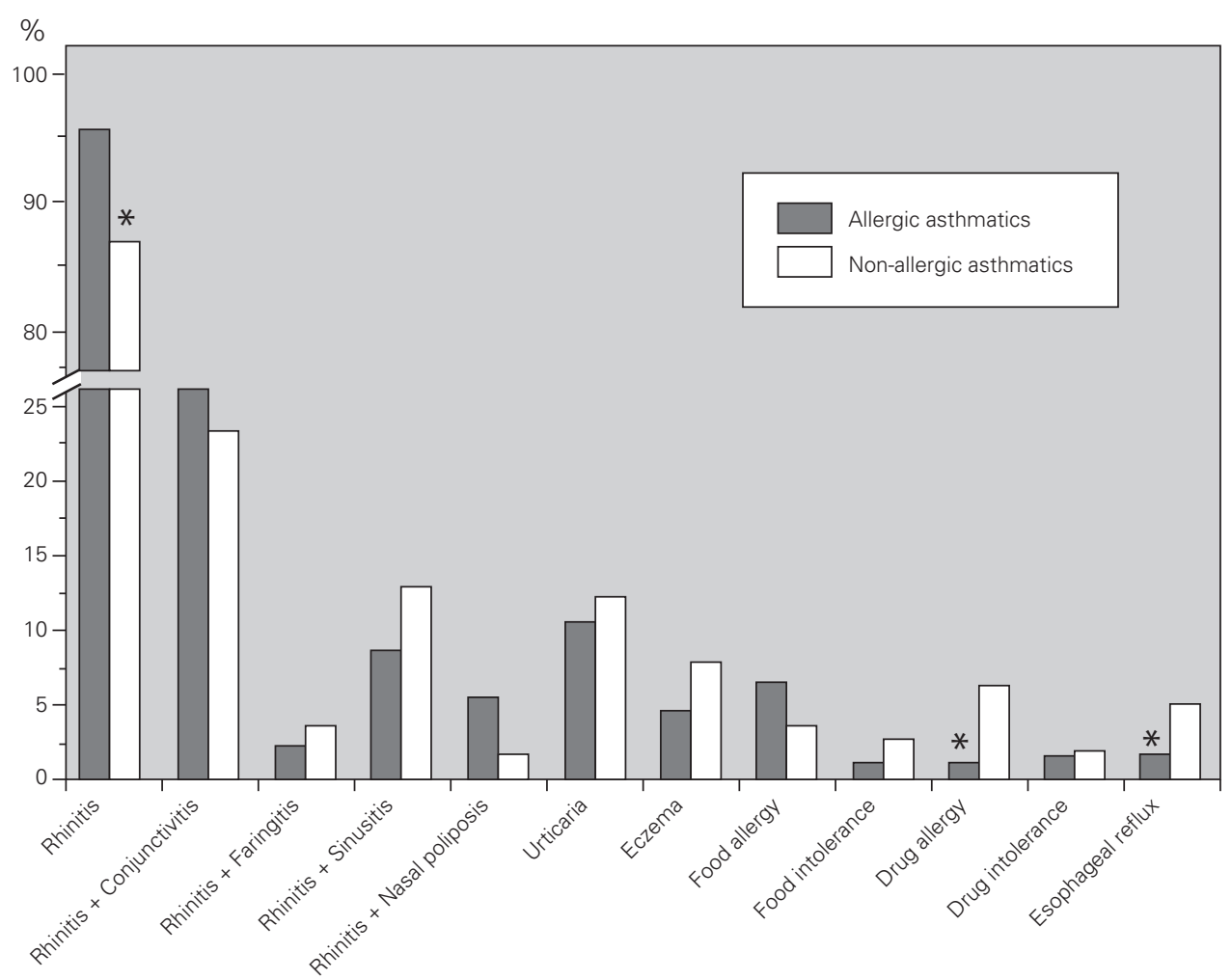

Figure 3.-Main co-morbidities associated with bronchial asthma. Allergic (dark columns) asthmatic patients had a higher prevalence of rhinitis, whereas non-allergic (light columns) asthmatic patients had a higher prevalence of drug allergy and oesophageal reflux.

Bronchial asthma is frequently associated with rhinosinusal involvement (even in non-allergic patients) and with other allergic diseases. We studied the main co-morbidities present in our asthmatic population and observed that the great majority of the asthmatic patients had rhinitis and conjunctivitis. There were differences between both groups, with non-allergic asthmatics having a lower prevalence of rhinitis, but a higher prevalence of drug allergy and oesophageal reflux as compared to allergic asthmatics (fig. 3).

As expected, total serum IgE was significantly higher in allergic asthmatics than in non-allergic asthmatics (median 205 [3-5000 kU/L] vs 27 [ $<2-653 \mathrm{kU} / \mathrm{L}]$, $p<0.001)$. There was no correlation between total $\lg E$ levels and features of allergic sensitisations (wheal size on the skin prick tests or specific lgE levels).

\section{Sensitisations in allergic asthma}

In allergic asthmatic patients, the frequency of sensitisations evaluated by skin prick testing was $65.5 \%$ for grass pollen, $62.9 \%$ for mites, $62.6 \%$ for cereal pollen, $49.3 \%$ for tree pollen, $49.3 \%$ for weed pollen, $30 \%$ for dog dander, $21.9 \%$ for moulds and fungi, and $15.3 \%$ for cat dander (fig. 4). The percentage of monosensitised allergic asthmatics was $12 \%$, with $6.5 \%$ of asthmatics being monosensitised to mites, $2.5 \%$ to grass pollen and $2.2 \%$ to weed pollen. There were no asthmatic patients monosensitised to cereal, dog dander or moulds. The frequency of sensitisation evaluated by specific IgE was slightly different but was directly correlated with that from skin prick tests (table II).

The major allergen sensitisers, as evaluated by skin prick testing and specific lgE, were grasses (Lolium perenne, Phleum pratense, Dactylis glomerata and Poa pratensis), cereals (Secale cereale, Triticum sativum and Avena sativa), mites (Dermatophagoides pteronyssinus and Dermatophagoides farinae), trees (Olea europea and Platanus acerifolia), the weed Parietaria judaica and the mould Alternaria alternata. A very interesting observation was that, in spite of similar prevalence of these major allergens, there were significant differences in terms of the class/level of sensitisation as measured by wheal size and specific lgE values (fig. 5). There was no correlation between the wheal size of the prick tests or the pattern of allergen sensitisation and the severity of the disease.

\section{Rural versus urban asthmatics}

As environmental factors, such as air pollution, are thought to play a part in sensitisation, we then com- 
Figure 4.-Cutaneous reactivity to aeroallergens in allergic asthmatics. Allergen sensitisation was evaluated by skin prick test with a battery of the most common aeroallergens in the local flora, as well as mites, moulds and animal dander.

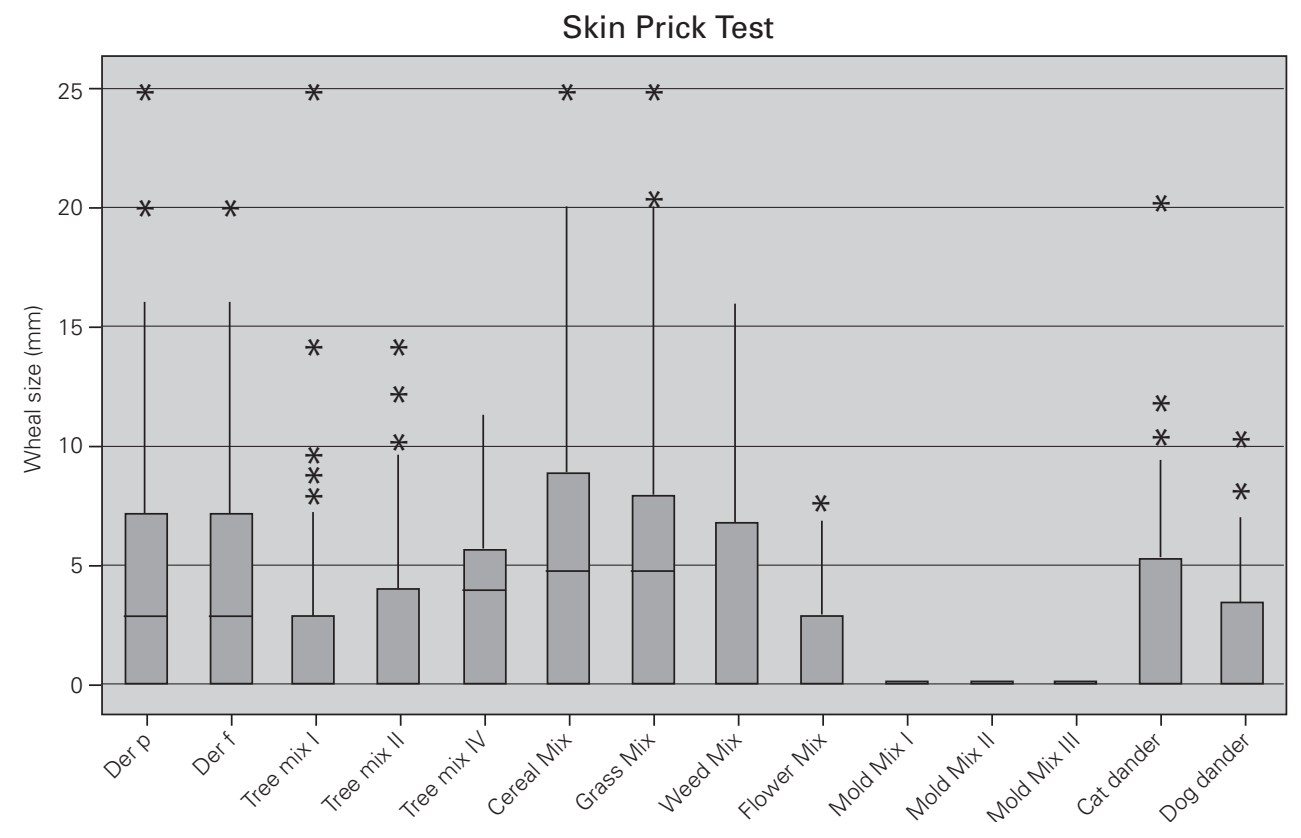

pared several features between urban and rural asthmatic patients. Our population included 141 urban and 182 rural allergic asthmatic patients. The samples were paired for age and gender.

The sensitisation profile was significantly different between urban- and rural-based asthmatic patients, with more rural than urban asthmatics being sensitised to tree pollen and less to moulds and fungi $\left(\chi^{2}\right.$ test, $50.3 \%$ vs $36.1 \% p=0.017$ and $7 \%$ vs $22.1 \% p<0.001$, respectively). However, the level of sensitisation was similar for all the allergens ( $p>0.05$ Mann Whitney $U$ test). Furthermore, there were no differences regarding severity of asthma between rural and urban allergic asthmatics and the same was valid for non-allergic asthmatic patients.

\section{DISCUSSION}

Asthma is a major public-health problem that is increasing in prevalence in most developed countries. Data regarding prevalence and sensitisation profiles in bronchial asthma are scarce in Portugal. Given its heterogeneous nature, it is important to thoroughly characterise bronchial asthma in order to improve treatment measures.

Thirty percent of our asthmatic patients were non-allergic. This proportion is analogous to that found in similar studies with hospital-based recruitment ${ }^{10}$, and is in accordance to the relative prevalence of non-allergic asthma, considered to vary between 10 and $30 \%{ }^{11}$.
Table II

Sensitisation profile in the allergic asthmatic patients

\begin{tabular}{lcc}
\hline \multicolumn{1}{c}{ Allergens } & Skin prick test $(\mathrm{n}, \%)$ & Specific lgE $(\mathrm{n}, \%)$ \\
\hline Cat dander & $101(36.3 \%)$ & $32(15.3 \%)$ \\
Cereal pollen & $174(62.6 \%)$ & $110(52.6 \%)$ \\
Dog dander & $83(30 \%)$ & $27(12.9 \%)$ \\
Grass pollen & $182(65.5 \%)$ & $123(58.9 \%)$ \\
Mites & $175(62.9 \%)$ & $123(58.9 \%)$ \\
Molds and fungi & $61(21.9 \%)$ & $4(1.9 \%)$ \\
Tree pollen & $137(49.3 \%)$ & $99(47.4 \%)$ \\
Weed pollen & $137(49.3 \%)$ & $116(55.5 \%)$ \\
\hline
\end{tabular}

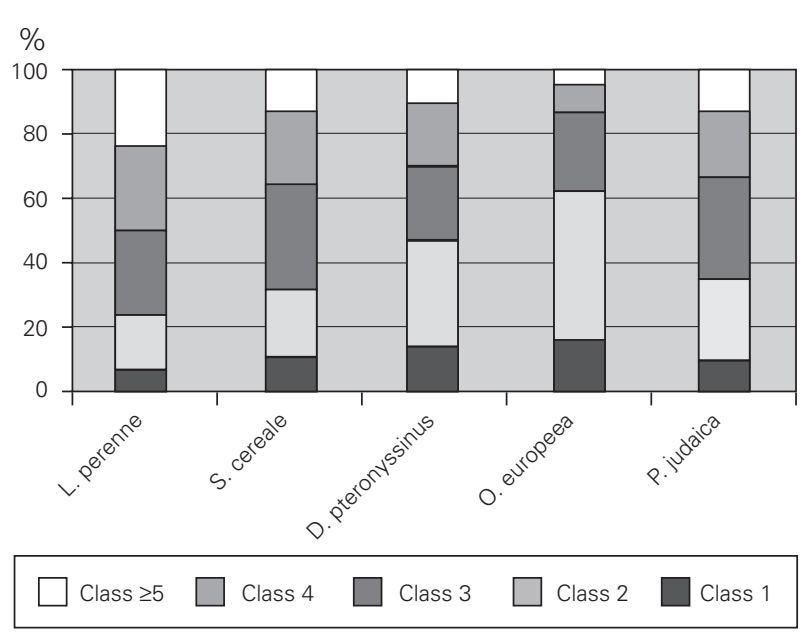

Figure 5.-Degree of sensitisation to aeroallergens in allergic asthmatic patients. The degree of sensitisation, as analysed by specific lgE levels, was different between the major aeroallergens. 
In the present study, we observed that non-allergic asthmatic patients were older, predominantly female, and had a higher prevalence of drug allergy and oesophageal reflux, but a lower prevalence of rhinitis than allergic asthmatics. A previous French study aiming at describing clinical similarities and differences between allergic and non-allergic asthmatics also found an association between non-allergic asthma and older age, and female sex. However, in contrast to this study, we did not find differences in terms of FEV1 values between allergic and non-allergic asthma ${ }^{10}$. This difference may be attributed to different study designs, namely in terms of severity and treatment of asthmatic patients included.

Asthma and rhinitis frequently occur concomitantly, with a reported prevalence of up to $100 \%$ in those with allergic asthma ${ }^{12}$. In the ECRHS, an association between asthma and rhinitis was observed even in non-atopic individuals ${ }^{13}$. In our study, there were significantly more allergic than non-allergic asthmatic patients with concurrent rhinitis. This may be due to the fact that many of the allergic asthmatic patients develop allergic rhinitis early in life and later develop bronchial asthma ${ }^{13}$. The importance of having concurrent rhinitis is highlighted by the key concept that has emerged in recent years that rhinitis and asthma should be viewed as disorders of a single airway. Having co-morbid allergic rhinitis is a marker for the presence of more-difficult-to-control asthma and worsened asthma outcomes ${ }^{4}$.

It is possible that the prevalence of co-morbid allergic rhinitis in this retrospective study was underestimated, because the diagnosis of allergic rhinitis was restricted to that recorded in medical records. Many people with allergic rhinitis self-manage the condition with over-the-counter products, do not seek a physician's help, or indeed do not recognize allergic rhinitis as a condition needing treatment. However, it must be borne in mind that all asthmatic patients included in this study were specifically asked about symptoms of rhinitis and anterior rhinoscopy was carried out in all of them.

Some patients with asthma have chronic cough as their main, if not only, symptom. Curiously, in our study, we found significantly more non-allergic than allergic asthmatic patients with cough-variant asthma. This cannot be explained in terms of severity of the underlying asthma since there were no differences between allergic and non-allergic patients in this regard. It may be due to the fact that significantly more non-allergic asthmatic patients had gastro-esophageal reflux. Alternatively, nonasthmatic patients may have a lower threshold for triggering cough reflex receptors in the bronchial mucosa.
In the literature non-allergic asthmatics characteristically have a later onset of symptoms with a more severe clinical course of the disease than those with allergic asthma. This is in clear contrast with the present study. This difference could be related to the fact that our patients were classified by asthma severity at the first appointment but many of them were not treatment-naïve at that time.

In our population, major allergic sensitisers included the graminae family (grass and cereal pollens) and mites. This is in agreement with a previous study that analysed aeroallergen sensitisation in the paediatric population of Cova da Beira, and which observed a similar pattern of sensitisation with grasses, Olea europea, Parietaria judaica as the most representative sensitisers ${ }^{14}$. The lberian study of aeroallergen sensitisation in allergic rhinitis reported similar results with common polysensitisation, involving both mites and pollens ${ }^{15}$, without significant differences between the coastal and inland areas.

In the present study we provide for the first time data on the magnitude of sensitisations for the major allergens in Portugal. Interestingly enough we show that there are significant differences in terms of the level of sensitisation as measured by specific IgE classes. In spite of similar prevalence within the major allergens, a particular species or family seems to be the main responsible agent for the symptoms, as happens with the grass pollen Lolium perenne in contrast to Olea europea. Accurate identification of the specific cause of allergic asthma is important to implement avoidance measures.

In terms of allergen sensitisation in urban- and rural-based asthmatic patients, we found a higher prevalence of tree pollen allergy in the rural context and a higher prevalence of sensitisation to moulds in the city. This may be due to the fact that city dwellings are more humid and tree pollen sources (Olea trees) are more densely present in the countryside, thereby locally increasing the allergen load. In contrast, in a similar study involving patients with allergic rhinitis in the Cova da Beira area, the prevalence of sensitisation to grasses, weeds and Olea europea was higher in the urban group ${ }^{16}$. In urban areas, pollen grains can become coated with fuel residues and combustion products, and this binding of pollen to diesel exhaust particles may modulate the allergenic epitopes and increase their allergenici$t^{17}{ }^{17}$. Our findings, however, may be related to the relative size of sensitiser particles, as our patients included essentially patients with bronchial asthma in contrast with the other patients, which involved essentially patients with allergic rhinitis.

One limitation of our study is that the study sample of adults with asthma was drawn from a special- 
ized allergy practice, and may differ significantly from the general population of asthmatic patients, since asthmatics treated by specialists appear to have more severe asthma than those seen by general practitioners.

In summary, in this study, rhinitis was more frequently associated with allergic than with non-allergic asthma, but drug allergy and oesophageal reflux were more associated with non-allergic asthma. In addition, the two types of asthma could not be differentiated in terms of severity of clinical symptoms.

The major allergens in the area were grass pollen, cereal pollen, mites and Olea europea. Monosensitisation was uncommon and it mainly included mites.

Urban and Rural based asthmatics had different sensitisation profiles, but could not be differentiated in terms of severity of their disease.

\section{ACKNOWLEDGEMENTS}

The authors would like to thank all the volunteers without whom this study would have been impossible, the administrative staff from the Hospital Centre and $A$. Raposo for invaluable help with the data base.

O.L. is the recipient of a fellowship from the Portuguese Foundation for Science and Technology (FCT) (BD16448/2004).

\section{REFERENCES}

1. Sennhauser FH, Braun-Fahrlander C, Wildhaber JH. The burden of asthma in children: a European perspective. Paediatr Respir Rev. 2005;6:2-7.

2. Borrego LM, Cesar M, Leiria-Pinto P, Rosado-Pinto JE. Prevalence of asthma in a Portuguese countryside town: repercussions on absenteeism and self-concept. Allergol Immunopathol (Madr). 2005,33:93-9.

3. Johansson SG, Hourihane JO, Bousquet J, BruijnzeelKoomen C, Dreborg S, Haahtela T, et al. A revised nomencla- ture for allergy. An EAACl position statement from the EAACl nomenclature task force. Allergy. 2001;56:813-24.

4. Jeffery PK, Haahtela T. Allergic rhinitis and asthma: inflammation in a one-airway condition. BMC Pulm Med. 2006;6 Suppl 1:S5.

5. Global Strategy for Asthma Management and Prevention. In. Bethesda: National Institutes of Health; 2002.

6. National Asthma Education and Prevention Program. Expert panel report. Guidelines for the diagnosis and management of asthma: update on selected topics. J Allergy Clin Immunol. 2002;110:S141-219.

7. Worldwide variation in prevalence of symptoms of asthma, allergic rhinoconjunctivitis, and atopic eczema: ISAAC. The International Study of Asthma and Allergies in Childhood (ISAAC) Steering Committee. Lancet. 1998;351:1225-32.

8. Worldwide variations in the prevalence of asthma symptoms: the International Study of Asthma and Allergies in Childhood (ISAAC). Eur Respir J. 1998;12:315-35.

9. Variations in the prevalence of respiratory symptoms, self-reported asthma attacks, and use of asthma medication in the European Community Respiratory Health Survey (ECRHS). Eur Respir J. 1996;9:687-95.

10. Romanet-Manent S, Charpin D, Magnan A, Lanteaume A, Vervloet D. Allergic vs nonallergic asthma: what makes the difference? Allergy. 2002;57:607-13.

11. Humbert M, Menz G, Ying S, Corrigan CJ, Robinson DS, Durham SR, Kay AB. The immunopathology of extrinsic (atopic) and intrinsic (non-atopic) asthma: more similarities than differences. Immunol Today. 1999;20:528-33.

12. Linneberg A, Henrik Nielsen N, Frolund L, Madsen F, Dirksen $A$, Jorgensen $T$. The link between allergic rhinitis and allergic asthma: a prospective population-based study. The Copenhagen Allergy Study. Allergy. 2002;57:1048-52.

13. Leynaert B, Neukirch C, Kony S, Guenegou A, Bousquet J, Aubier M, Neukirch F. Association between asthma and rhinitis according to atopic sensitization in a population-based study. J Allergy Clin Immunol. 2004;113:86-93.

14. Loureiro G, Rabaca MA, Blanco B, Andrade S, Chieira C, Pereira C. Aeroallergens sensitization in an allergic paediatric population of Cova da Beira, Portugal. Allergol Immunopathol (Madr). 2005;33:192-8.

15. Pereira C, Valero A, Loureiro C, Davila I, Martinez-Cocera C, Murio $C$, et al. Iberian study of aeroallergens sensitisation in allergic rhinitis. Allerg Immunol (Paris). 2006;38:186-94.

16. Loureiro G, Rabaca MA, Blanco B, Andrade S, Chieira C, Pereira C. Urban versus rural environment - any differences in aeroallergens sensitization in an allergic population of Cova da Beira, Portugal? Allerg Immunol (Paris). 2005;37:187-193.

17. Parnia S, Brown JL, Frew AJ. The role of pollutants in allergic sensitization and the development of asthma. Allergy. 2002; 57:1111-7. 